\title{
Clinical and ultrastructural spectrum of diffuse lung disease associated with surfactant protein $\mathrm{C}$ mutations
}

\author{
Donatella Peca ${ }^{1}$, Renata Boldrini ${ }^{2}$, Jan Johannson ${ }^{3}$, Joseph T Shieh ${ }^{4}$, Arianna Citti $^{2}$, Stefania Petrini ${ }^{1}$, \\ Teresa Salerno ${ }^{5}$, Salvatore Cazzato $^{6}$, Raffaele Testa ${ }^{7}$, Francesco Messina ${ }^{8}$, Alfredo Onofri ${ }^{9}$, Giovanna Cenacchi $^{10}$, \\ Per Westermark ${ }^{11}$, Nicola Ullmann ${ }^{5}$, Paola Cogo $^{12}$, Renato Cutrera ${ }^{5}$ and Olivier Danhaive $e^{\star, 4,13}$
}

Genetic defects of surfactant metabolism are associated with a broad range of clinical manifestations, from neonatal respiratory distress syndrome to adult interstitial lung disease. Early therapies may improve symptoms but diagnosis is often delayed owing to phenotype and genotype variability. Our objective was to characterize the cellular/ultrastructural correlates of surfactant protein C (SP-C) mutations in children with idiopathic diffuse lung diseases. We sequenced SFTPC - the gene encoding SP-C - SFTPB and ABCA3, and analyzed morphology, ultrastructure and SP expression in lung tissue when available. We identified eight subjects who were heterozygous for SP-C mutations. Median age at onset and clinical course were variable. None of the mutations were located in the mature peptide-encoding region, but were either in the pro-protein BRICHOS or linker C-terminal domains. Although lung morphology was similar to other genetic surfactant metabolism disorders, electron microscopy studies showed specific anomalies, suggesting surfactant homeostasis disruption, plus trafficking defects in the four subjects with linker domain mutation and protein misfolding in the single BRICHOS mutation carrier in whom material was available. Immunolabeling studies showed increased proSP-C staining in all cases. In two cases, amyloid deposits could be identified. Immunochemistry and ultrastructural studies may be useful for diagnostic purposes and for genotype interpretation.

European Journal of Human Genetics (2015) 23, 1033-1041; doi:10.1038/ejhg.2015.45; published online 18 March 2015

\section{INTRODUCTION}

Pediatric diffuse parenchymal lung diseases (pDLD) are rare disorders with various etiologies, characterized by chronic or progressive gas exchange impairment and multifocal or diffuse infiltrates. Mutations in several surfactant-related genes have been increasingly recognized as causes of $\mathrm{pDLD}{ }^{1}$ these genes encode different types of protein: intrinsic surfactant peptides (surfactant protein B (SP-B) and C $(\mathrm{SP}-\mathrm{C})^{2}$ ), transmembrane transporters (ATP-binding cassette protein A3 $\left.(\mathrm{ABCA} 3)^{3}\right)$, gene expression regulators (thyroid transcription factor 1 (TTF1)), receptors (granulocyte macrophage colony stimulating factor receptor subunits $\mathrm{a}$ and $\mathrm{b}(\operatorname{CSFR} 2 \alpha$ and $\beta)$ ), accounting in part for the extreme diversity of the clinical spectrum. SP-C is a small hydrophobic peptide, which, in association with SP-B, has a key role in surface film formation and stability at the alveolar gas-liquid interface. ${ }^{4}$ The $S P-C$ gene, also designated $S F T P C$, is located at the 8p21 locus, spanning $3.5 \mathrm{~kb}$, is composed of six exons, and encodes a 191 or 197 amino-acid (aa) apoprotein depending upon alternative splicing, which subsequently traffics through the endoplasmic reticulum (ER) and the Golgi and undergoes cleavage of $\mathrm{N}$ and $\mathrm{C}$ terminus residues in multivesicular bodies (MVBs), leading to formation of the 35 aa mature SP-C peptide. ${ }^{5}$ This peptide interacts with SP-B and phospholipids inside the lamellar bodies (LBs) to form bioactive surfactant. The $\mathrm{N}$ terminus domain is involved in cell trafficking and the $\mathrm{C}$ terminus domain harbors the BRICHOS domain, a chaperone preventing aggregation of the hydrophobic mature peptide during posttranscriptional processes. SP-B and ABCA3 deficiencies are autosomal recessive diseases presenting as neonatal respiratory distress syndrome (RDS) typically fatal within the first months of age (although certain ABCA3 mutations may lead to later onset disease $^{6}$ ). SP-C mutations are mostly mono-allelic, either sporadic or inherited, and are expressed in a dominant fashion in $\sim 50 \%$ of cases. Clinical onset varies from birth to advanced adulthood, with variable severity and outcome. ${ }^{7}$ Complex molecular mechanisms account in part for the phenotypic diversity of the disease. Environmental factors also have a key role in modulating the disease course, as carriers of the same mutation in a single pedigree may show a broad variability of onset and presentation. ${ }^{8,9}$ Lung infections often trigger or complicate the course of pDLD in SP-C mutation carriers, especially in infantile age. In the context of an ongoing research project aimed at determining the contribution of rare and common variants of surfactant-related genes in pDLD, we report the clinical features and molecular and histological findings in eight subjects heterozygous for SFTPC rare variants.

\footnotetext{
${ }^{1}$ Research core laboratories, Bambino Gesu' Children's Hospital, Rome, Italy; ${ }^{2}$ Division of Anatomopathology, Bambino Gesu' Children's Hospital, Rome, Italy; ${ }^{3}$ Department of Neurobiology, Care sciences and Society, Karolinska Institutet, Huddinge, Sweden; ${ }^{4}$ Department of Pediatrics, University of California San Francisco Benioff Children's Hospital, San Francisco, CA, USA; ${ }^{5}$ Department of Pediatrics, Bambino Gesu' Children's Hospital, Rome, Italy; ${ }^{6}$ Division of Pediatric Pulmonology, S. Orsola-Malpighi University Hospital, Bologna, Italy; ${ }^{7}$ Division of Pediatric intensive Care, Santobono Hospital, Naples, Italy; ${ }^{8}$ Division of Neonatology, Villa Betania Hospital, Naples, Italy; ${ }^{9}$ Division of Pediatric Intensive Care, Bambino Gesu' Children's Hospital, Rome, Italy; ${ }^{10}$ Division of Clinical Pathology, S. Orsola-Malpighi University Hospital, Bologna, Italy; ${ }^{11}$ Department of Immunology, Genetics and Pathology, Uppsala University, Uppsala, Sweden; ${ }^{12}$ Department of Pediatric Cardiology and Cardiac Surgery, Bambino Gesu' Children's Hospital, Rome, Italy; ${ }^{13}$ Department of Medical and Surgical Neonatology, Bambino Gesu' Children's Hospital, Rome, Italy

*Correspondence: Dr O Danhaive, Department of Pediatrics, University of California San Francisco, San Francisco General Hospital, 1001 Potrero Avenue, Mailstop 6E, San Francisco, CA 94110, USA. Tel: +1 415206 8361; Fax: +1 415206 3686; E-mail: danhaiveo@peds.ucsf.edu
}

Received 16 May 2013; revised 10 February 2015; accepted 11 February 2015; published online 18 March 2015 


\section{MATERIALS AND METHODS}

Subjects

From 2005 to 2012, infants and children with a history of unexplained persistent or progressive diffuse lung disease based on clinical signs (abnormal auscultation, cough, dyspnea, hypoxemia) with an onset between birth and 18 years plus evidence of diffuse parenchymal lung disease were referred to our center for genetic testing. Exclusion criteria consisted of acute/chronic airway infections, cystic fibrosis, aspiration pneumonia, immune deficiencies, primary ciliary dyskinesia, tuberculosis and allergic bronchopulmonary aspergillosis. Parental consent was obtained when applicable. We sequenced SFTPC, SFTPB and $A B C A 3$, plus other genes in selected cases based on family history and clinical findings (online supplement). Patient no. 7 was referred to our center as a young adult but was included in this series as she had respiratory symptoms since 1 year of age. The study was conducted in compliance with the hospital internal review board and research ethics committee.

\section{DNA analysis}

We performed PCR-based mutation analysis of the SFTPC, SFTPB and ABCA3 genes by Sanger's technique. All coding exons were amplified by PCR with primers synthesized for the coding and flanking regions of each gene using the human genome sequences ENSG00000168484 (SFTPC), ENSG00000168878 (SFTPB) and ENSG00000167972 (ABCA3), and were sequenced bidirectionally. ${ }^{10}$ Amino-acid sequences were expressed following the NCBI NP_001165881 (proSP-C) and NP_001080.2 (ABCA3) reference sequences. Familial DNA was sequenced when available, and rare variants were tested in 100 alleles, and in the 1000 Genomes Project phase1 (1000G, http://www.1000genomes.org) and the NHLBI Grand Opportunity Exome Sequencing Project (ESP, http://evs.gs. washington.edu) databases. The impact of predicted aa changes on protein structure and function was assessed by SIFT ${ }^{11}$ and PolyPhen-2 (http://genetics. bwh.harvard.edu/pph2). Amino-acid conservation was assessed by sequence comparison in 28 vertebrate species. The SFTPC variants were submitted to the Leiden Open Variation Database (LOVD) public database (http://www.lovd.nl); identification numbers are provided in Table 1. For previously described variants, we also provided the identification number in the dbSNP public database when available.

\section{Optical microscopy}

Sections of formaldehyde-fixed paraffin-embedded lung tissue were labeled with 1:100 mouse anti SP-B antibody (Lab Vision, Fremont, CA, USA) or 1:400 polyclonal rabbit anti proSP-C (Millipore, Temecula, CA, USA) ${ }^{12}$ and detected with peroxidase-coupled secondary antibodies (Dako, Glostrup, Denmark) after staining with hematoxylin-eosin; normal lung samples were obtained from three age-matched subjects (3-, 12- and 18-month-old) deceased from a nonpulmonary cause. Lung tissue was analyzed for amyloid by polarized light optical microscopy after Congo Red staining. ${ }^{13}$ When frozen tissue was available, we performed confocal fluorescence microscopy after dual immunolabeling with proSP-C, an ER marker (PDI) and a LB and lysosome marker (LAMP3) (see online supplement).

\section{Transmission electron microscopy}

Samples from cases no. 1-3 and 6 were obtained at the main institution following similar procedures, fixed in Karnovsky's fixative, postfixed in $1 \%$ $\mathrm{OsO} 4$ and embedded in EMbed-812. Ultrathin sections stained with uranyl acetate and lead citrate were examined with a Zeiss EM CENTRA 100 microscope (Carl Zeiss, Oberkochen, Germany). Case no. 6 was obtained in another institution and processed differently: fresh bronchoalveolar lavage (BAL) samples were centrifuged at $800 \mathrm{RPM}$; cell pellets were resuspended in $2.5 \%$ glutaraldehyde in $0.1 \mathrm{~m}$ cacodylate buffer, postfixed in $1 \% \mathrm{OsO} 4$, dehydrated in graded ethanol and embedded in araldite. Ultrathin sections stained with uranyl acetate and lead citrate were examined with a Philips 410 microscope (Philips, Eindhoven, the Netherlands).

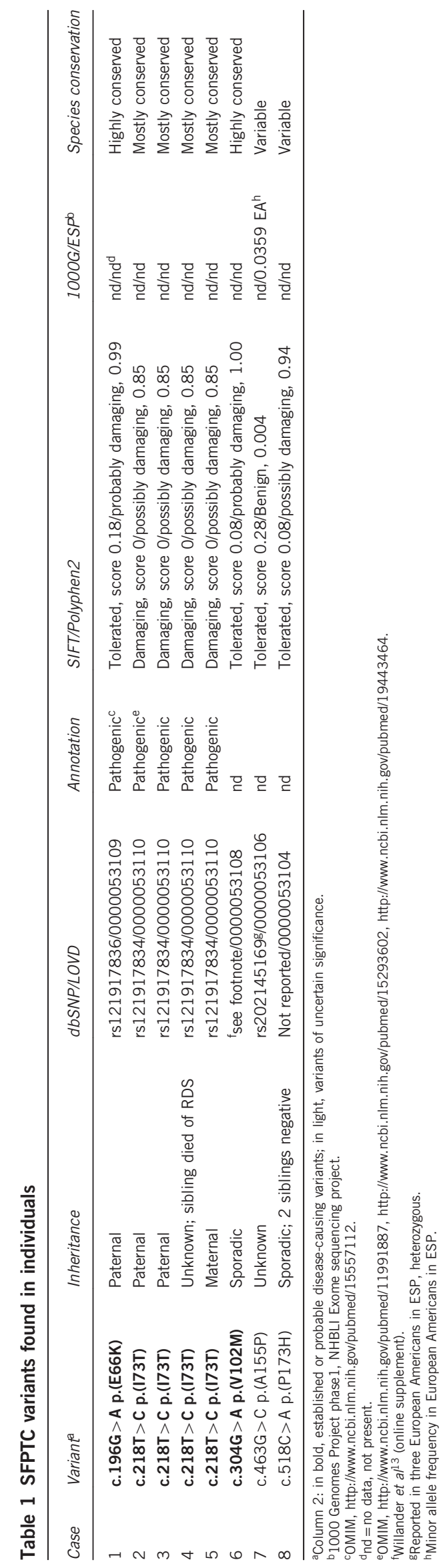




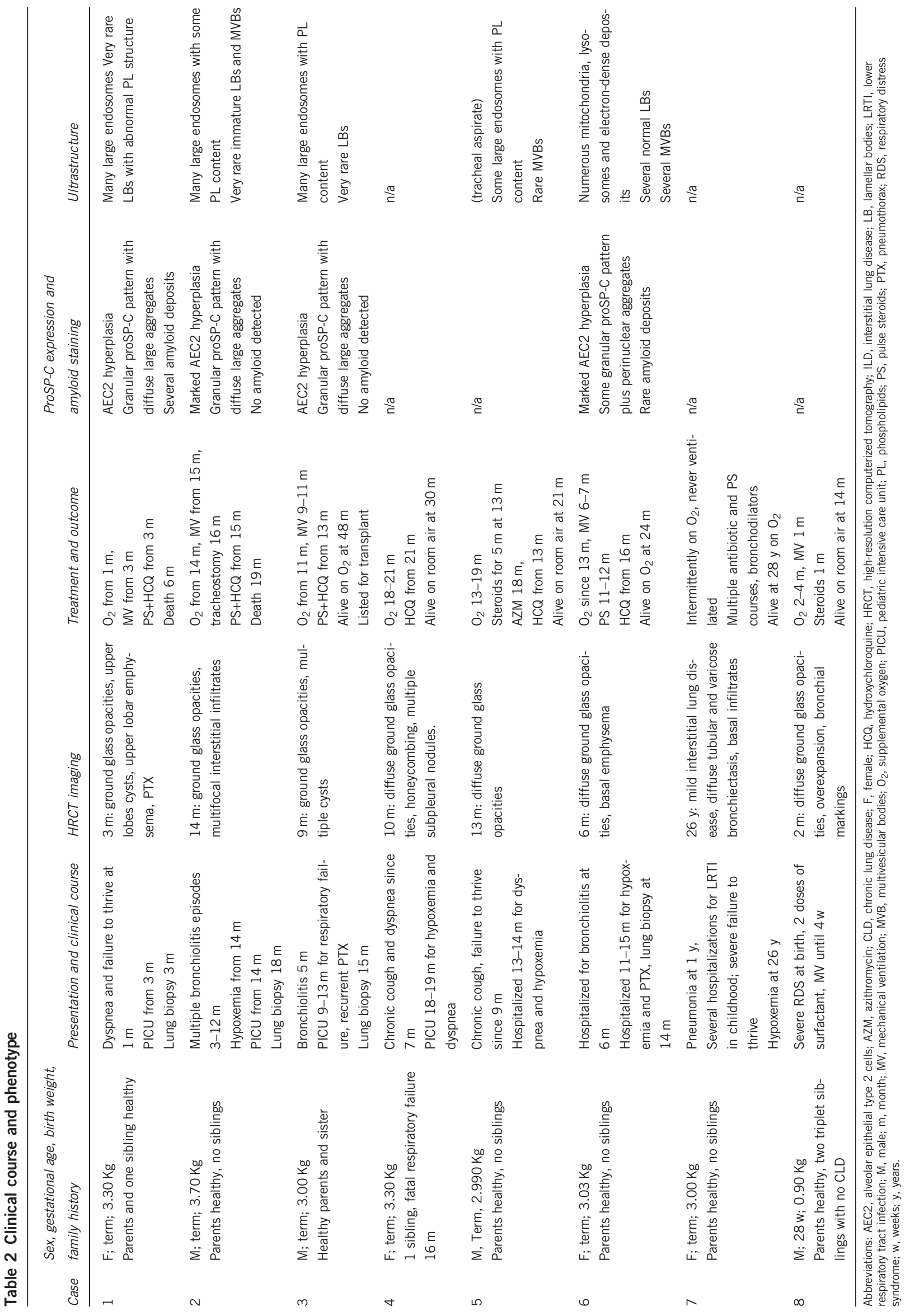




\section{RESULTS}

\section{Clinical features}

(Table 2) All infants were Caucasian and of Italian origin except patient no. 4, who was of Romanian origin. They were born at term except patient no. 8 at 28 weeks of gestation, all with birth weight appropriate for gestational age (median $3.01 \mathrm{Kg}$; range 0.90-3.70). Four were males and four females. One had a family history of a sibling with fatal lung disease at 16 months. Median age at onset was 5.5 months (range $0-12$ ). All except patient no. 8 showed significant postnatal failure to thrive. One infant (case no. 8) was a 28 -week preterm triplet who presented with unexpectedly severe RDS compared with the two siblings, requiring multiple surfactant administrations, mechanical ventilation until 32 weeks and supplemental oxygen until 1 month corrected gestational age, whereas the other two siblings were only on CPAP for $<3$ weeks. The proband's subsequent course was normal, with a follow-up of 15 months. The other seven cases were asymptomatic in early infancy and presented with respiratory symptoms from 1-12 months, five of which after viral bronchiolitis. Lung high-resolution computed tomography (HRCT) performed at a median age of 9 months (except case no. 7) showed ground glass opacities plus a combination of small and large cysts, subpleural nodules, lobar emphysema, air leaks and/or focal infiltrates. Case no. 7's prominent feature was multiple bronchiectasis. ${ }^{14}$ Two infants died at 6 and 8 months, respectively; among survivors, two were on home oxygen therapy at 25 and 48 months with one enlisted for lung transplant, two were on room air with recurrent exacerbations requiring oxygen supplementation, and two were stable with no symptoms.

\section{Molecular genetics}

Of the 88 subjects of the cohort, eight harbored an SFTPC rare coding variant (Table 1); in four (no. 2, 4, 5 and 6) the mutation was inherited from one parent, who was asymptomatic. Patient no. 1 carried the p.(Glu66Lys) mutation, previously reported to cause neonatal respiratory failure; ${ }^{15}$ in addition, we found the unreported ABCA3 synonymous c.1383 G>A coding variant (p. $($ Val461 =), LOVD:0000055959) of unclear clinical significance. Cases no. 2-5 carried the p.(Ile73Thr) mutation, the most frequently reported with a broad spectrum of manifestations from neonatal RDS to adult ILD. ${ }^{7,8,16-23}$ Patient no. 6 carried the c.304G $>$ A variation leading to the p.(Val102Met) aa change, previously reported without clinical description. ${ }^{13}$ In patient no. 7 , the c. $463 \mathrm{G}>\mathrm{C}$ variation was identified in exon 5, causing the p.(Ala155Pro) aa change, previously published as a case report. ${ }^{14}$ Patient no. 8 carried the previously unreported c.518C $>$ A variation leading to the p.(Pro173His) aa change. The new variants reported here were not present in 100 control alleles, and were neither found in 1000G nor in ESP except for patient no. 7 (c.463G $>$ C variant, MAF 0.0359).

\section{Anatomopathology}

The four cases in whom a lung biopsy was performed (no. 1-3 and 6) showed a similar pattern of SP-B-positive alveolar epithelial type II cells hyperplasia, alveolar spaces filled with SP-B-positive desquamated epithelial cells and alveolar macrophages, and interstitial thickening with fibroblast proliferation, corresponding to desquamative interstitial pneumonia (or pulmonary alveolar proteinosis in older literature).

\section{Immunohistochemistry}

In controls, immunostaining for proSP-C showed a focal cytoplasmic expression adjacent to LBs, which appear as clearer, outlined vesicles at the apical pole. ${ }^{9}$ In subjects with linker domain mutations (cases no. 1 , no. 2 and no. 3), there was increased staining for ProSP-C, which appeared to be localized largely to cytoplasmic vesicles similar to those identified by others as endosomes in the same p.(Glu66Lys) mutation. ${ }^{15}$ In the BRICHOS mutant (case no. 6), there was also markedly increased proSP-C staining with some denser staining lining the nucleus, similar to the misfolded protein response pattern described in a $\Delta$ exon4 (BRICHOS) mutagenesis experiment ${ }^{24}$ (Figure 1).

\section{Dual channel immunofluorescence}

In the patient carrying the linker domain p.(Glu66Lys) mutation (case no. 1), confocal microscopy with co-labeling of SP-B and proSP-C showed a much stronger signal than SP-B compared with control, suggesting proSP-C overexpression (not shown). ProSP-C was only limitedly co-expressed with DPI in the p.(Glu66Lys) mutant as well as in the control lungs. However, the mutant showed a stronger co-expression of proSP-C with LAMP3 than the control, with a pattern of large cytoplasmic vesicles (Supplementary Figure S1, online supplement).

\section{Polarized light microscopy}

In cases no. 1 and 6 (Figure 2), small amyloid deposits with typical staining properties were identified in the interstitium, in association with macrophages, not found in lung tissue from control subjects. We attempted immunolabeling experiments with antibodies specifically targeted to the C-terminal propeptide and to the BRICHOS domain, but the small size of amyloid deposits precluded identification of immunoreactive material (not shown).

\section{Transmission electron microscopy}

(Figure 3) Compared with control, Cases no. 1, no. 2 and no. 3 (linker domain mutants) showed numerous, large organelles containing amorphous material and scarce electron-dense phospholipid structures likely corresponding to early endosomes. ${ }^{25}$ Only a few MVBs and immature LBs with altered pseudomyelin structure could be observed in these subjects, the content of which is secreted in the alveolar lumen (case no. 1, Supplementary Figure S2, online supplement), suggesting a profound disruption of surfactant homeostasis. A similar pattern could be observed in an alveolar epithelial type 2 cell isolated from bronchoalveolar lavage in patient no. 5 , also a linker domain mutant. In contrast, patient no. 6 (BRICHOS mutant) showed a hyperplastic ER compared with control, perinuclear electron-dense cytoplasmic aggregates, abundant lysosomes, and some MVBs and immature LBs showing pseudomyelin structures, albeit less than in control.

\section{DISCUSSION}

SP-C mutations are a significant and probably underestimated cause of diffuse lung disease at various ages. Prevalence in pDLD series, as in ours, varies from 8 to $17 \% .^{1,3,6,7,19,26}$ SP-C mutations have been described in term ${ }^{15,27}$ or late preterm infants with severe RDS, although this presentation is more typical of SP-B and ABCA3 biallelic mutations. ${ }^{2}$ The typical presentation of SP-C defects consists of dyspnea, cough or wheezing with an onset between 2 and 12 months of age, gradual cyanosis and failure to thrive. ${ }^{8,20,23,28}$ In this paper the youngest subject, a 28 -week preterm triplet carrying the p.(Pro173His) mutation was identified by comparing RDS severity with his two siblings with wild-type SP-C alleles. Conversely, in the literature, SP-C mutations have been found in adults with familial idiopathic pulmonary fibrosis ${ }^{29}$ and in adults $14-68$ year-old in one pedigree, ${ }^{30}$ 

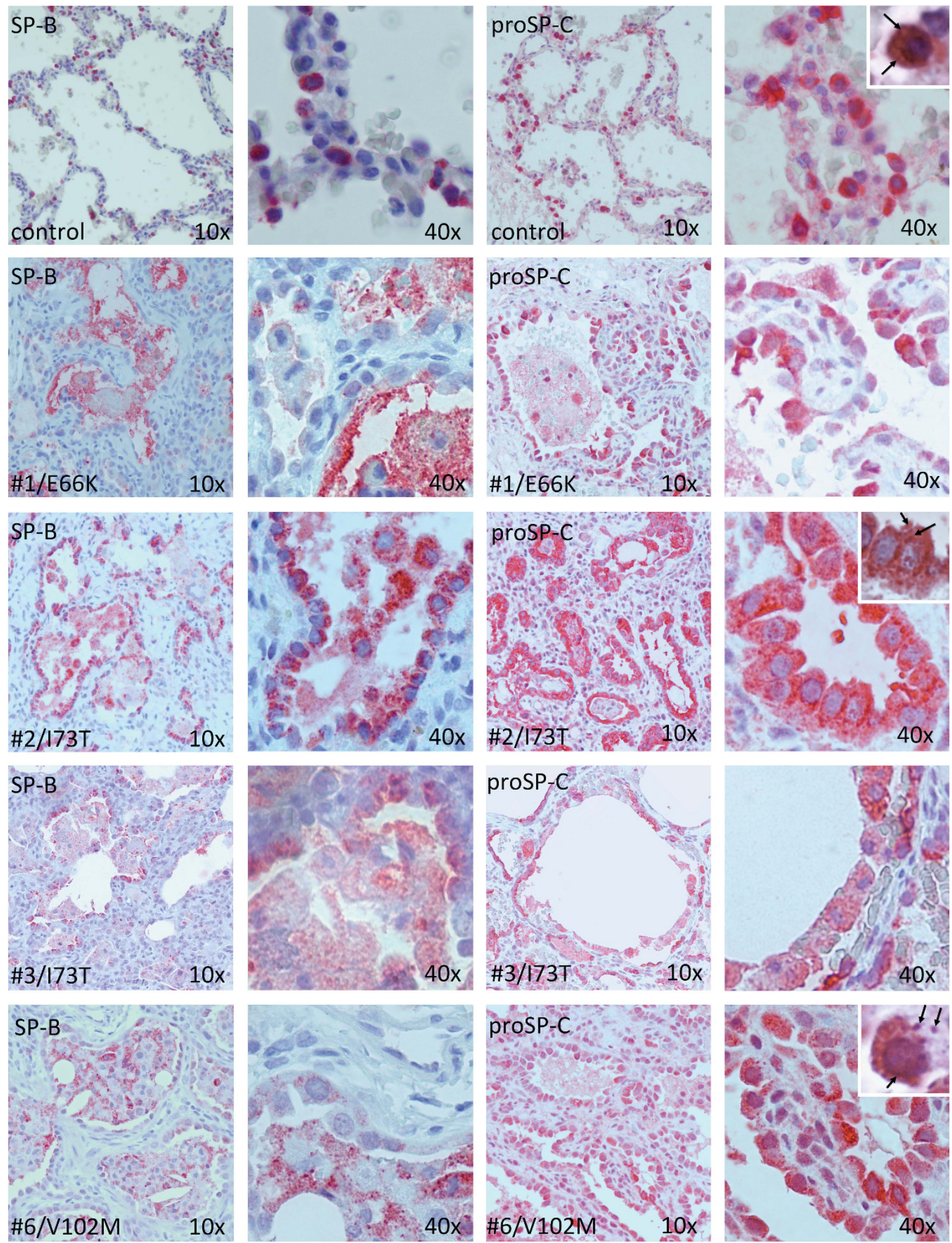

Figure 1 Lung tissue morphology and surfactant protein immunohistochemistry. Immunohistochemistry staining for SP-B (columns 1 and $2,10 \times$ and $40 \times$ magnification, respectively) and pro-SP-C (columns 3 and 4) in a 3-month-old control (row 1), three linker domain mutants (row 2-4) and one BRICHOS domain mutant (row 5). Alveolar epithelial type 2 cell hyperplasia is present in all four cases compared with control. In control, proSP-C is expressed in the cytoplasm surrounding clearer-appearing lamellar bodies (row 1, insert, arrows). In cases no. 1-3, proSP-C is diffusely overexpressed in the cytoplasm with a granular pattern (row 3, insert, arrows). In case no. 6, proSP-C is overexpressed with a perinuclear pattern and some scattered aggregates (row 5, insert, arrows). Counterstaining with hematoxylin-eosin. 

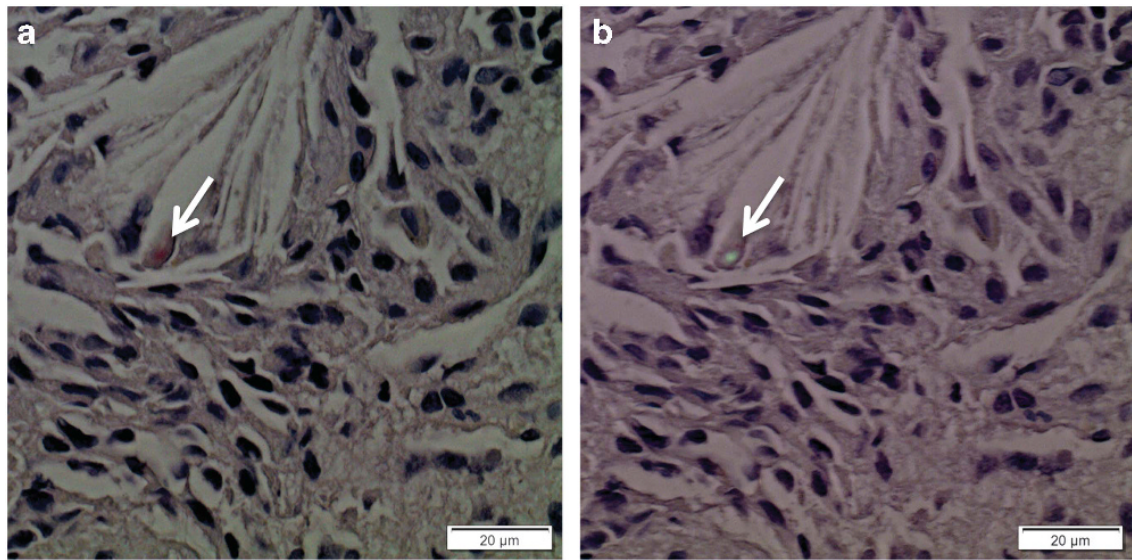

Figure 2 Amyloid detection. (a) Congo red staining of case no. 6 lung specimen showing a small Congo-positive deposit in the interstitium, near cholesterol clefts (arrow). (b) Same specimen viewed under polarized light optical microscopy, the deposit showing green birefringence indicating amyloid (arrow)

suggesting that environmental factors or individual genetic background are significant determinants.

Steroids, hydroxychloroquine and azithromycin are commonly used in SP-C-related pDLD. Azithromycin has anti-amyloid properties, ${ }^{31}$ and hydroxychloroquine affects intracellular proSP-C processing; 32 hence these drugs may possibly have selective effects on specific mutations, although there are currently no clinical data available to support this concept. All of our subjects received one or more of these interventions at various points in their disease course, some before the biopsy was obtained, so it is difficult to know if these treatments modified the histologic or ultrastructural appearance in any manner.

The common radiology characteristic in our cases was ground glass opacities, present in all cases except no. 7. As median age at HRCT was 9 months except for case no. 7 (26 years), age may be a determining factor, although we cannot exclude mutation severity or other factors. In the literature, chest radiograms and/or HRCT show diffuse ground glass opacities in neonates, ${ }^{15}$ whereas in older infants and children, interstitial thickening associated with lung hyperinflation, intraparenchymal/supleural cysts, honeycombing, subpleural nodules ${ }^{8,33}$ or bronchiectasis ${ }^{14}$ are more prominent. These patterns are not different from those observed in other genetic surfactant deficiencies. ${ }^{34}$ Infiltrates and air leaks are frequent complication in acute exacerbations. ${ }^{17}$ Focal or diffuse reticulonodular patterns, centrolobular and subpleural nodules and cystic lesions are described in adults. ${ }^{29,30}$

We did not observe specific differences in lung histology between our cases, in whom lung biopsy was obtained at a median age of 6 months. We observed a combination of DIP with features of PAP, a pattern common to most genetic surfactant deficiencies in infants. ${ }^{1}$ In the literature, older children and adults with SP-C mutations display patchy fibrotic lesions of usual interstitial pneumonia or diffuse nonspecific interstitial pneumonia, in combination with nodular septa thickening and clustered cystic lesions, ${ }^{29,30}$ suggesting lung tissue morphology is more age-specific rather than disease-specific.

However, by TEM we observed significant anomalies in LB number and appearance in all cases examined, indicating altered surfactant composition/ structure. These alterations are distinct from those observed in SP-B or ABCA3 deficiency $y^{3,6}$ and appear specific in both our observations and the literature. ${ }^{15,28}$ In SP-C-deficient mice, LBs and extracellular surfactant are similar to controls, ${ }^{35}$ suggesting that the human disease is not primarily caused by SP-C haploinsufficiency itself. However, even though human SP-C mutations are mono-allelic and both the wild-type and the mutated alleles are transcribed, mature
SP-C peptide is not (or barely) detected in lung tissue and bronchoalveolar lavage fluid. ${ }^{17,36}$ Overall, these findings and data suggest that surfactant deficiency, owing to a toxic gain-of-function mechanism affecting LB formation and surfactant synthesis, may contribute in part to the pathogenesis in human. In addition, in the three carriers of linker domain mutations in whom lung biopsy was performed (cases no. 1-3), we observed numerous large endosomes. Similar, albeit smaller, vesicles were observed in the other linker mutant (case no. 5), although the material was obtained by BAL and processed differently, hence may not be strictly comparable. Although very few TEM data have been published on human subjects with SP-C mutations, similar vesicles in one linker mutation case were shown by immunogold staining to colocalize with proSP-C, suggesting a trafficking defect. ${ }^{15}$ Conversely in the single BRICHOS domain mutation carrier analyzed by TEM (case no. 6), we found hyperplastic ER and perinuclear electron-dense aggregates, which, if confirmed in other cases, could reflect misfolded protein-related cell toxicity. ${ }^{37}$

Although bi-allelic mutations have been reported exceptionally, ${ }^{13,20}$ loss-of-function is not a prominent mechanism in human SP-C deficiency, which is typically caused by mono-allelic mutations. SP-C null mutant mice are viable, showing that SP-C is not critical for survival. Hence, different pathophysiological processes, depending on distinct types of gene defects, are involved in human SP-C mutation-associated lung disease and, likely, account for this phenotypic heterogeneity.

Mutations in the linker (non-BRICHOS) sequence (aa59-89) of the C-terminal peptide exert their pathogenic effect by inducing aberrant intracellular trafficking of proSP-C, which eludes cleavage and accumulates in endosomes and MVBs. ${ }^{25}$ In cases no. $1-3$, we observed large proSP-C-positive vesicles in type II cell cytoplasm by immunohistochemistry. Confocal immunofluorescence microscopy in one case (no. 1) showed partial colocalization of proSP-C and LAMP3, a marker expressed in MVBs and LBs, confirming abnormal accumulation of the uncleaved propeptide in these organelles. Overall, our observations in human subjects are compatible with the model of proSP-C misprocessing, accumulation in MVBs, aberrant secretion and reuptake in the endosomal/lysosomal pathway derived from animal and in vitro studies ${ }^{24,25}$ but are less clear-cut and may be of limited clinical use, likely because of the coexistence of a wild-type and a mutated SFTPC allele, both expressed to a certain degree and resulting in a mixed phenotype. 

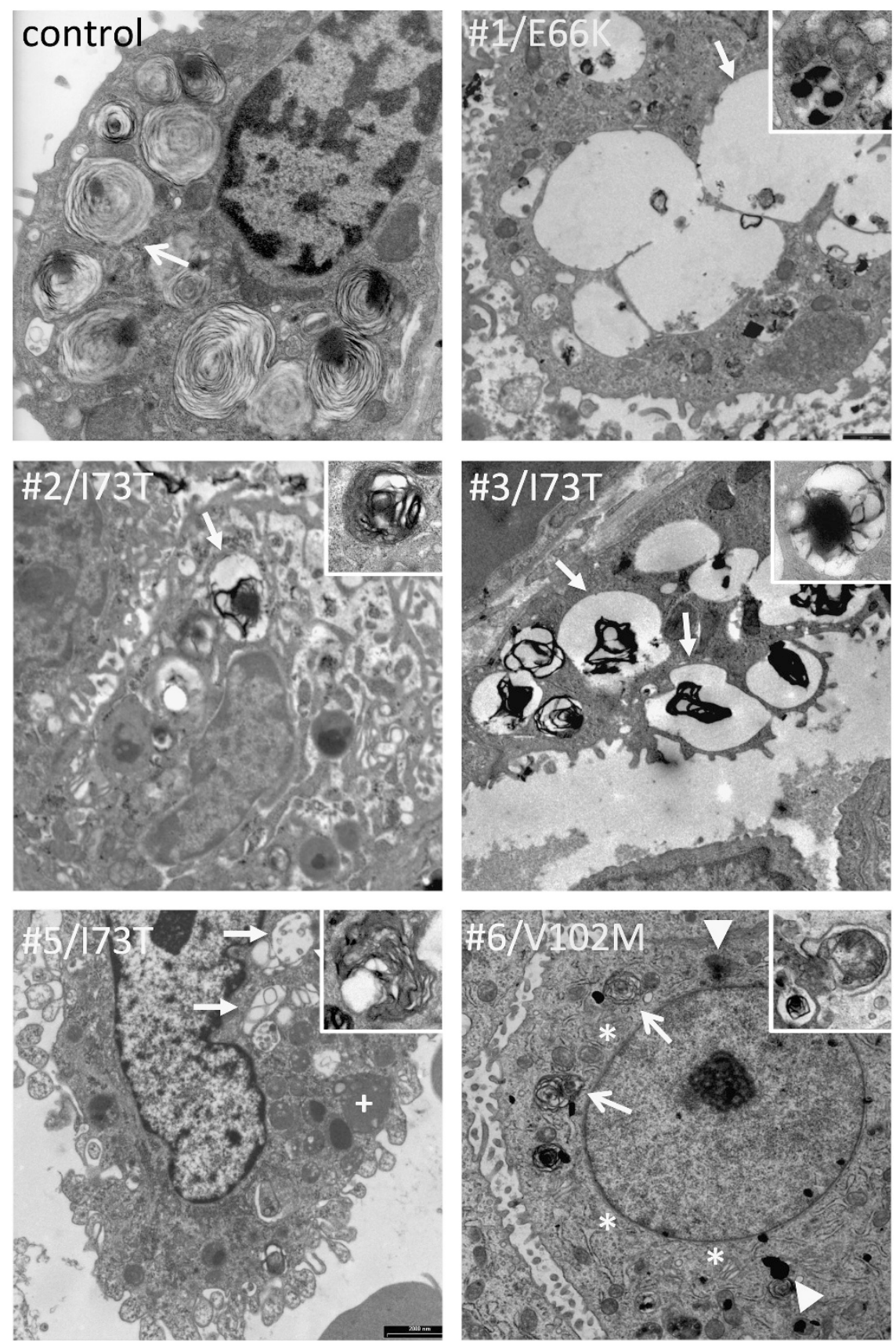

Figure 3 Type II cell ultrastructure. Type II cell sections in a control subject, four linker domain mutation carriers (cases no. 1-3 and 5) and one BRICHOS mutation carrier (no. 6). Tissue was obtained by autopsy for control and live biopsy for cases no. 1-3 and 6; cells were obtained by bronchoalveolar lavage for case no. 5. In control, numerous mature lamellar bodies with pseudomyelin are present (arrow). Cases no. 1-4 show numerous, large coalescing endosomes with scarce amorphous content (arrow), some lysosomes (+), and few multivesicular bodies (inserts). Case no. 6 shows hypertrophic endoplasmic reticulum $\left(^{*}\right)$ compared with control, some cytoplasmic electron-dense deposits (block arrows) and several multivesicular bodies (arrows) with disorganized phospholipid membranes and amorphous content (insert).

The majority of mutations reported in human affect the highly conserved BRICHOS domain (aa90-197), common to proSP-C and other proteins associated with dementia, and bone and gastric cancer. The mature SP-C peptide is highly hydrophobic and tends to form $\beta$-sheets aggregates and amyloid fibrils in vitro. ${ }^{13,38}$ In fact, overexpression of the mature SP-C peptide in transgenic mice leads to neonatal lethality. ${ }^{38}$ The BRICHOS domain functions as a chaperone, preventing aggregation and amyloid formation. In vitro mutagenesis and transgenic mice studies demonstrated that BRICHOS domain mutations lead to SP-C misfolding, accumulation in the ER, amyloid formation, disruption of the ubiquitin-proteasome system, activation of the apoptosis cascade and type II cell death. ${ }^{3,24,39,40}$ The proSP-C perinuclear expression pattern observed in case no. 6 may reflect peptide segregation and aggregation in the ER, but confirmatory confocal immunofluorescence studies with a specific ER marker could not be performed in this case owing to the lack of frozen tissue, and 
more cases would be necessary to confirm these speculations. We could demonstrate the presence of amyloid in the interstitium in the same case, further supporting this mechanism. However, amyloid was also detected in patient no. 1, suggesting that protein misfolding also occurs to some degree in linker domain mutants.

However, these conclusion should be balanced by the limited number of cases with lung tissue available, and by the fact that, as in most pathology studies based on human cases, it is difficult to establish which findings are part of the underlying disease process per se, which are a response to the underlying mechanism of disease, and which might be the result of interventions (such as mechanical ventilation) or duration or severity of disease.

The five subjects with linker domain mutations carried known pathogenic variants, four of which were p.(Ile73Thr). Although linker domain mutations have been reported to have a less severe clinical expression than BRICHOS mutation in some series, ${ }^{7,17}$ our own data and the literature do not necessarily support this concept. We found three BRICHOS domain rare variants in our series. No other SFTPB, $A B C A 3$ or NKX2.1 mutations were present in these subjects. The p.(Vall02Met) variant (case no. 6) is predicted to be deleterious in silico by both algorithms and is mostly conserved among species; its pathogenic role is supported by histologic and ultrastructural findings including amyloid deposits; ${ }^{13}$ it was also reported in another patient with neonatal-onset respiratory failure (16, online supplement). The p.(Phe173His) variant (case no. 8), unreported so far, appears damaging on PolyPhen-2 but not on SIFT, and is also less conserved among species (Table 1), making its pathogenicity questionable. The more severe clinical course in the proband compared with the non-mutated siblings supports mutation pathogenicity, but prematurity (as a potential cause of lung disease) and the favorable outcome may suggest it as a benign variant. The role of the p.(Arg155Pro) variant (case no. 7) is also less clear, as this missense mutation shows a low damaging probability and has been reported in three subjects of European descent $(0.04 \%)$ in the ESP database. As no lung tissue was available for phenotype correlation and the parents did not consent for genetic testing, we cannot affirm that this variant is responsible for the subject's lung disease. The relevance of the associated synonymous ABCA3 mutation observed in patient no. 1 is uncertain. Compound genotypes associating p.(Ile73Thr) and an ABCA3 mutation have been reported in infants with $\mathrm{pDLD}^{19}$ and in adults with late-onset ILD, suggesting that a mono-allelic $\mathrm{ABCA} 3$ mutation may act as disease modifier in SP-C defects. ${ }^{21}$

\section{CONCLUSION}

This case series illustrates the vast array of symptoms and outcomes associated with SP-C deficiency, and shows correlations between known and new genotypes and lung ultrastructure, supporting the importance of lung biopsy including TEM analysis for accurate diagnosis. Broncho-alveolar lavage may represent an alternative, less invasive diagnostic procedure than lung biopsy if validated in future studies. As this clinical variability is only partially supported by molecular and genetic mechanisms, future research should focus on determining individual genotypes through genomic approach to expand the understanding of genetic-clinical correlations and interactions with other surfactant-related genes.

This study was limited by the number of cases and by the lack of mutagenesis studies susceptible to better establish disease mechanism correlations. As for any rare disorder, only large collaborative initiatives may yield the sufficient power of validating new diagnostic and therapeutic approaches.

\section{CONFLICT OF INTEREST}

The authors declare no conflict of interest.

\section{ACKNOWLEDGEMENTS}

We are grateful to Dr Paola Carrera, Clinical Molecular Biology Laboratory, Università Vita-Salute San Raffele, Milan, Italy for SFTPC sequencing in patient no. 5. This publication was supported by an unrestricted grant by the Chiesi Foundation, Parma, Italy.

1 Deutsch GH, Young LR, Deterding RR et al: Diffuse lung disease in young children: application of a novel classification scheme. Am J Respir Crit Care Med 2007; 176 $1120-1128$.

2 Somaschini M, Nogee LM, Sassi I et al: Unexplained neonatal respiratory distress due to congenital surfactant deficiency. J Pediatr 2007; 150: 649-653, 653 e641.

3 Shulenin S, Nogee LM, Annilo T, Wert SE, Whitsett JA, Dean M: ABCA3 gene mutations in newborns with fatal surfactant deficiency. N Engl J Med 2004; 350: 1296-1303.

4 Blanco O, Perez-Gil J: Biochemical and pharmacological differences between preparations of exogenous natural surfactant used to treat Respiratory Distress Syndrome: role of the different components in an efficient pulmonary surfactant. Eur $J$ Pharmacol 2007; 568: 1-15.

5 Brasch F, Ten Brinke A, Johnen G et al: Involvement of cathepsin H in the processing of the hydrophobic surfactant-associated protein $\mathrm{C}$ in type II pneumocytes. Am J Respir Cell Mol Biol 2002; 26: 659-670.

6 Bullard JE, Wert SE, Whitsett JA, Dean M, Nogee LM: ABCA3 mutations associated with pediatric interstitial lung disease. Am J Respir Crit Care Med 2005; 172 1026-1031.

7 Guillot L, Epaud R, Thouvenin G et al: New surfactant protein C gene mutations associated with diffuse lung disease. J Med Genet 2009; 46: 490-494.

8 Abou Taam R, Jaubert F, Emond $S$ et al: Familial interstitial disease with 173T mutation: a mid- and long-term study. Pediatr Pulmonol 2009; 44: 167-175.

9 Thomas AQ, Lane K, Phillips J 3rd et al: Heterozygosity for a surfactant protein C gene mutation associated with usual interstitial pneumonitis and cellular nonspecific interstitial pneumonitis in one kindred. Am J Respir Crit Care Med 2002; 165 1322-1328.

10 McBee AD, Wegner DJ, Carlson CS et al: Recombination as a mechanism for sporadic mutation in the surfactant protein-C gene. Pediatr Pulmonol 2008; 43: 443-450.

11 Sim NL, Kumar P, Hu J, Henikoff S, Schneider G, Ng PC: SIFT web server: predicting effects of amino acid substitutions on proteins. Nucleic Acids Res 2012; 40: W452-W457.

12 Vorbroker DK, Profitt SA, Nogee LM, Whitsett JA: Aberrant processing of surfactant protein C in hereditary SP-B deficiency. Am J Physiol 1995; 268: L647-L656.

13 Willander $\mathrm{H}$, Askarieh $\mathrm{G}$, Landreh $\mathrm{M}$ et al: High-resolution structure of a BRICHOS domain and its implications for anti-amyloid chaperone activity on lung surfactant protein C. Proc Natl Acad Sci USA 2012; 109: 2325-2329.

14 Salerno T, Peca D, Rossi FP, Menchini L, Danhaive O, Cutrera R: Bronchiectasis and severe respiratory insufficiency associated with a new surfactant protein $\mathrm{C}$ mutation. Acta Paediatr 2012; 102: e1-e2.

15 Stevens PA, Pettenazzo A, Brasch F et al: Nonspecific interstitial pneumonia, alveolar proteinosis, and abnormal proprotein trafficking resulting from a spontaneous mutation in the surfactant protein C gene. Pediatr Res 2005; 57: 89-98.

16 Nogee LM, Dunbar AE 3rd, Wert S, Askin F, Hamvas A, Whitsett JA: Mutations in the surfactant protein $C$ gene associated with interstitial lung disease. Chest 2002; 121: 20S-21S.

17 Thouvenin G, Abou Taam R, Flamein F et al: Characteristics of disorders associated with genetic mutations of surfactant protein C. Arch Dis Child 2010; 95: 449-454.

18 Brasch F, Griese M, Tredano M et al: Interstitial lung disease in a baby with a de novo mutation in the SFTPC gene. Eur Respir J 2004; 24: 30-39.

19 Bullard JE, Nogee LM: Heterozygosity for ABCA3 mutations modifies the severity of lung disease associated with a surfactant protein C gene (SFTPC) mutation. Pediatr Res 2007; 62: 176-179.

20 Cameron HS, Somaschini M, Carrera P et al: A common mutation in the surfactant protein C gene associated with lung disease. J Pediatr 2005; 146: 370-375.

21 Crossno PF, Polosukhin VV, Blackwell TS et al: Identification of early interstitial lung disease in an individual with genetic variations in ABCA3 and SFTPC. Chest 2010; 137: 969-973.

22 Lawson WE, Grant SW, Ambrosini V et al: Genetic mutations in surfactant protein C are a rare cause of sporadic cases of IPF. Thorax 2004; 59: 977-980.

23 Percopo S, Cameron HS, Nogee LM, Pettinato G, Montella S, Santamaria F: Variable phenotype associated with SP-C gene mutations: fatal case with the I73T mutation. Eur Respir J 2004; 24: 1072-1073.

24 Mulugeta S, Nguyen V, Russo SJ, Muniswamy M, Beers MF: A surfactant protein C precursor protein BRICHOS domain mutation causes endoplasmic reticulum stress, proteasome dysfunction, and caspase 3 activation. Am J Respir Cell Mol Biol 2005; 32: $521-530$ 
25 Beers MF, Hawkins A, Maguire JA et al: A nonaggregating surfactant protein C mutant is misdirected to early endosomes and disrupts phospholipid recycling. Traffic 2011; 12: $1196-1210$

26 Nathan N, Taam RA, Epaud R et al: A national internet-linked based database for pediatric interstitial lung diseases: the French network. Orphanet J Rare Dis 2012; 7: 40 .

27 Soraisham AS, Tierney AJ, Amin HJ: Neonatal respiratory failure associated with mutation in the surfactant protein $C$ gene. J Perinatol 2006; 26: 67-70.

28 Hamvas A, Nogee LM, White FV et al: Progressive lung disease and surfactant dysfunction with a deletion in surfactant protein C gene. Am J Respir Cell Mol Biol 2004: 30: 771-776.

29 van Moorsel $\mathrm{CH}$, van Oosterhout MF, Barlo NP et al: Surfactant protein C mutations are the basis of a significant portion of adult familial pulmonary fibrosis in a dutch cohort. Am J Respir Crit Care Med 2010; 182: 1419-1425.

30 Ono S, Tanaka T, Ishida M et al: Surfactant protein C G100S mutation causes familial pulmonary fibrosis in Japanese kindred. Eur Respir J 2011; 38: 861-869.

31 Tucker S, Ahl M, Bush A, Westaway D, Huang X, Rogers JT: Pilot study of the reducing effect on amyloidosis in vivo by three FDA pre-approved drugs via the Alzheimer's APP 5' untranslated region. Curr Alzheimer Res 2005; 2: 249-254.

32 Beers MF: Inhibition of cellular processing of surfactant protein $C$ by drugs a ffecting intracellular pH gradients. J Biol Chem 1996; 271: 14361-14370.
33 Tredano M, Griese M, Brasch F et al: Mutation of SFTPC in infantile pulmonary alveolar proteinosis with or without fibrosing lung disease. Am J Med Genet A 2004; 126A: 18-26.

34 Young LR, Nogee LM, Barnett B, Panos RJ, Colby TV, Deutsch GH: Usual interstitial pneumonia in an adolescent with ABCA3 mutations. Chest 2008; 134: 192-195.

35 Glasser SW, Burhans MS, Korfhagen TR et al: Altered stability of pulmonary surfactant in SP-C-deficient mice. Proc NatI Acad Sci USA 2001; 98: 6366-6371.

36 Nogee LM, Dunbar AE 3rd, Wert SE, Askin F, Hamvas A, Whitsett JA: A mutation in the surfactant protein $\mathrm{C}$ gene associated with familial interstitial lung disease. $N$ Engl J Med 2001; 344: 573-579.

37 Mulugeta S, Maguire JA, Newitt JL, Russo SJ, Kotorashvili A, Beers MF: Misfolded BRICHOS SP-C mutant proteins induce apoptosis via caspase-4- and cytochrome c-related mechanisms. Am J Physiol Lung Cell Mol Physiol 2007; 293: L720-L729.

38 Conkright JJ, Na CL, Weaver TE: Overexpression of surfactant protein-C mature peptide causes neonatal lethality in transgenic mice. Am J Respir Cell Mol Biol 2002; 26: 85-90.

39 Zarbock R, Woischnik M, Sparr C et al: The surfactant protein C mutation A116D alters cellular processing, stress tolerance, surfactant lipid composition, and immune cell activation. BMC Pulm Med 2012; 12: 15.

40 Bridges JP, Wert SE, Nogee LM, Weaver TE: Expression of a human surfactant protein C mutation associated with interstitial lung disease disrupts lung development in transgenic mice. J Biol Chem 2003; 278: 52739-52746.

Supplementary Information accompanies this paper on European Journal of Human Genetics website (http://www.nature.com/ejhg) 